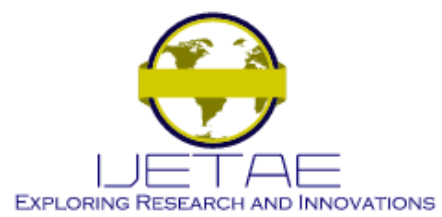

International Journal of Emerging Technology and Advanced Engineering

Website: www.ijetae.com (ISSN 2250-2459, ISO 9001:2008 Certified Journal, Volume 4, Issue 2, February 2014)

\title{
A Fault Analysis in Reversible Sequential Circuits
}

\author{
B. Anuradha ${ }^{1}$, S. Sivakumar ${ }^{2}$ \\ ${ }^{1}$ PG scholar, Assistant Professor,Department of ECE, Karpagam University, Coimbatore, Tamilnadu, India.
}

\begin{abstract}
In this paper, the researchers propose the design of reversible circuits using reversible gates. Reversible logic is implemented in reversible circuits. Reversible logic is mostly preferred due to less heat dissipation. Conservative logic gates can be designed in any sequential circuits and can be tested using two test vectors. The significance of proposed work lies in the design of reversible sequential circuits and their equivalent circuits for maximum fault coverage. The design of reversible sequential circuits using Toffoli gate and Peres gate is proposed in this literature. The design of Toffoli and Peres equivalent circuits is proposed first time in this literature, in order to achieve maximum fault coverage. The proposed Toffoli and Peres gates surpass the Fredkin gate and MXCQCA gate in terms of area, number of gates and timing. The simulation and coding is performed using cadence tool.

Keywords-Reversible logic, Peres gate, Toffoli gate, Feynman gate, latches.
\end{abstract}

\section{INTRODUCTION}

Reversible logic is one of the property in which there exists the one-one mapping between input and output. Reversible logic has the same number of inputs and outputs. It produces the output pattern for each input. Energy loss is avoided by reversible logic. Various scientists have undergone research on reversible logic like Landeuer's and Benett. Landauer has undergone research on irreversible logic where each bit of information generates KTln2 Joules of heat energy. Benett proved that KTln2 energy dissipation would not occur if a computation takes place in a reversible way. Reversible logic is suitable for many applications like Low Power Consumption, Quantum computers, Nano Technology and Speed improvement. Fanouts are not allowed in the reversible logic synthesis. Reversible logic synthesis differs from irreversible logic synthesis.

Various definitions can be formed using reversible logic. They are reversible function, reversible logic gate, garbage, Quantum cost, gate levels or logic depth, Flexibility and gate count. Reversible logic can be synthesized using Combinational and sequential logic. Most of the researches took place on combinational logic, but in Sequential logic require the memory cells and feedback in a circuit.

In this Paper, we propose a design of Reversible Sequential circuits using Toffoli gate and Peres gate. Toffoli equivalent circuit and Peres equivalent circuit are proposed in order to attain maximum fault coverage.
This paper is organized as follows: Section III presents the proposed Reversible Toffoli and Peres gate. Section IV describes the design of Reversible latches using Toffoli and Peres gate. Section V describes the design of Negative enable Reversible D latch using Toffoli and Peres gate. Section VI presents the design of master slave flipflops using Toffoli and Peres gate. Section VII demonstrates the design of Reversible DET flipflops using Toffoli and Peres gate. Section VIII presents the design of Toffoli equivalent circuit. Section IX describes the design of Peres equivalent circuit. Section $\mathrm{X}$ provides the results and conclusion.

\section{BACK GROUND}

A gate is said to be reversible if only it satisfies the function is bijective. It is mapping from input function to the output function.Few objectives need to satisfy the reversible circuit. There are number of gates; minimize the number of garbage outputs and Synthesis. Various researches had been studied in reversible logic. Feynman gate, New gate and Fredkin gate are used for reversible logic synthesis [3].

Piston and Frank introduced their reversible logic operations [4]. In reversible gates, any Boolean function can be utilized [5].

\section{A. Related work.}

Reversible logic is used for many applications like quantum computing. Algorithm is presented to synthesize reversible functions. In reversible functions, the algorithm was positive polarity reed Muller expansion to synthesize the function of Toffoli gates [6]. Few problems faced by reversible logic synthesis are fanouts not allowed and feedback from gate output to inputs is not allowed. CMOS implementations of reversible gates are proposed [7]. The designs of reversible sequential circuits are introduced to minimize the quantum cost and delay number of garbage outputs[12]. Various New reversible logic gates are introduced using Boolean expansion [12]. 


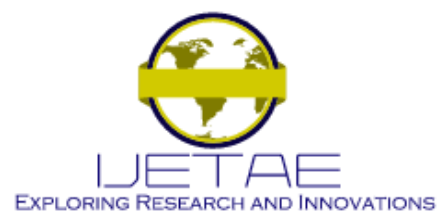

\section{International Journal of Emerging Technology and Advanced Engineering}

Website: www.ijetae.com (ISSN 2250-2459, ISO 9001:2008 Certified Journal, Volume 4, Issue 2, February 2014)

III. Proposed Reversible Toffoli And Peres Gates

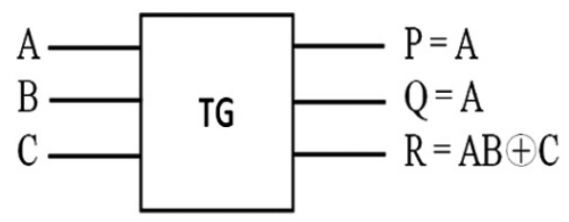

Figure 1: Toffoli gate

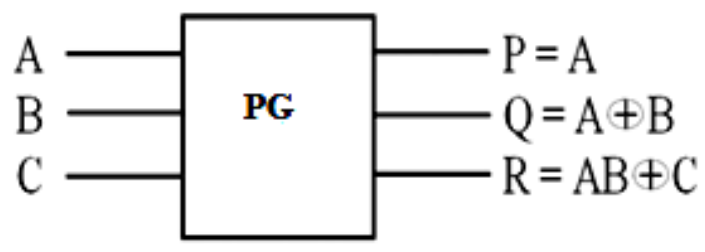

Figure 2: Peres gate

TABLE 1

TRUTH TABLE OF TOFFOLI GATE

\begin{tabular}{|l|l|l|l|l|l|}
\hline \multicolumn{3}{|c|}{ Input } & \multicolumn{3}{c|}{ Output } \\
\hline 0 & 0 & 0 & 0 & 0 & 0 \\
\hline 0 & 0 & 1 & 0 & 0 & 1 \\
\hline 0 & 1 & 0 & 0 & 1 & 0 \\
\hline 1 & 0 & 0 & 1 & 0 & 0 \\
\hline 1 & 0 & 1 & 1 & 0 & 1 \\
\hline 1 & 1 & 0 & 1 & 1 & 1 \\
\hline 1 & 1 & 1 & 1 & 1 & 0 \\
\hline
\end{tabular}

TABLE 2

TRUTH TABLE OF PERES GATE

\begin{tabular}{|l|l|l|l|l|l|}
\hline \multicolumn{3}{|c|}{ Input } & \multicolumn{3}{c|}{ Output } \\
\hline 0 & 0 & 0 & 0 & 0 & 0 \\
\hline 0 & 0 & 1 & 0 & 0 & 1 \\
\hline 0 & 1 & 0 & 0 & 1 & 0 \\
\hline 0 & 1 & 1 & 0 & 1 & 1 \\
\hline 1 & 0 & 0 & 1 & 1 & 0 \\
\hline 1 & 0 & 1 & 1 & 1 & 1 \\
\hline 1 & 1 & 0 & 1 & 0 & 1 \\
\hline 1 & 1 & 1 & 1 & 0 & 0 \\
\hline
\end{tabular}

Toffoli gate inverted by Tommaso Toffoli is one of the universal reversible logic gate. $\mathrm{A}, \mathrm{B}$, and $\mathrm{C}$ are the inputs to Toffoli gate and $\mathrm{P}, \mathrm{Q}, \mathrm{R}$ are the outputs. When two bits are set, it alters the third bit otherwise all remain the same. Peres gate has three inputs and outputs. Each input is mapped to the each output $(\mathrm{P}=\mathrm{A}, \mathrm{Q}=\mathrm{A}$ XOR $\mathrm{B} \mathrm{R}=\mathrm{AB}$ XOR C). It has the quantum cost of 4 .

IV. Design Of REVERSIBLE LATCHES USING TOFFOLI AND PERES GATE

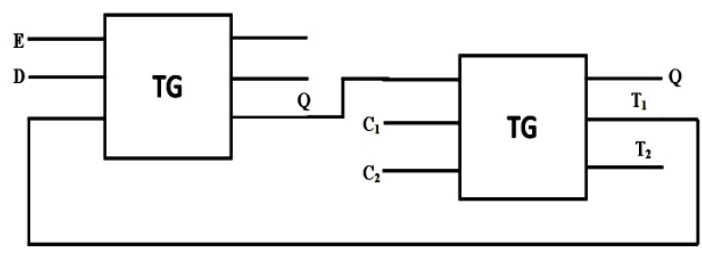

Figure 3: Design of Reversible D latch with Control signals using Toffoli gate

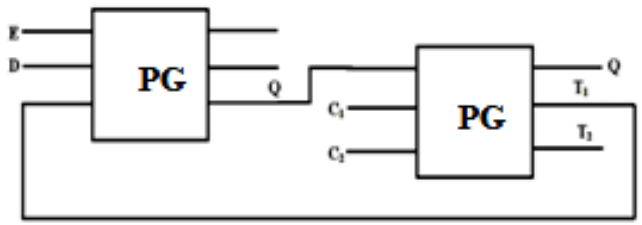

Figure 4: Design of Reversible D latch with Control signals using Peres gate 


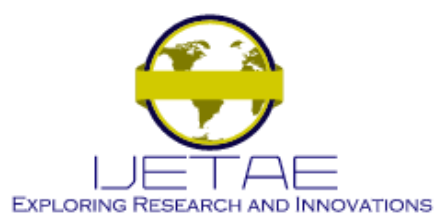

\section{International Journal of Emerging Technology and Advanced Engineering}

\section{Website: www.ijetae.com (ISSN 2250-2459, ISO 9001:2008 Certified Journal, Volume 4, Issue 2, February 2014)}

Enable e and $\mathrm{d}$ are the input signals to the $\mathrm{D}$ latch in Toffoli gate and $\mathrm{P}, \mathrm{Q}, \mathrm{R}$ are the output signals. When the enable is zero the value of $d$ is passed to the output $q$ and $r$. When the enable is one, the value of $d$ is passed to the output $\mathrm{p}$ and $\mathrm{q}$ (latch maintains the previous state of $\mathrm{q}$ ). Latch is tested with control signals $\mathrm{C} 1$, and $\mathrm{C} 2$, when $\mathrm{C} 1 \mathrm{C} 2$ $=01$, the latch works as fault free mode in Toffoli and Peres gate. When $\mathrm{C} 1 \mathrm{C} 2=00$ or 11 , the latch works as test mode in Toffoli and Peres gate. In both these modes, there is a shift in the output waveform and latch is tested for various modes of operations.

\section{Design Of Negative Enable Reversible D Latch USING TOFFOLI AND PERES GATE}

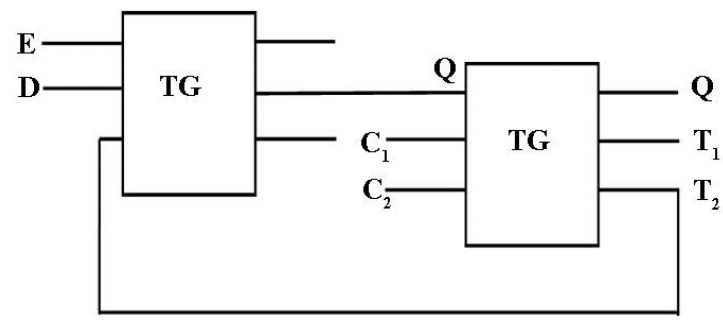

Figure 5: Design of Negative enable Reversible D latch using Toffoli gate

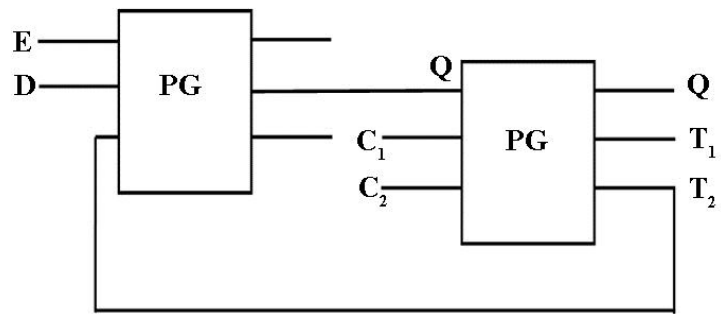

Figure 6: Design of Negative enable Reversible D latch Using Peres gate

Toffoli gate and Peres gate are tested in Negative D latch. The latch is tested with various values of enable, D signals latch works in normal mode or test mode depends upon the value of control signal.

\section{Design Of MASTER SlaVe D Flipflops Using TOFFOLI AND PERES GATE}

In Existing Literature, master slave flipflops are designed using Fredkin gate. In this paper, the researchers have proposed the design of master slave D flipflops using Toffoli and Peres gate.

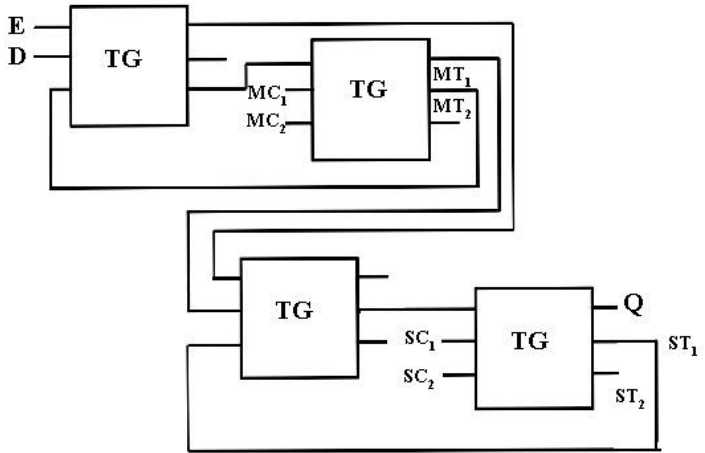

Figure 7: Design of Master slave D flipflops using Toffoli gate

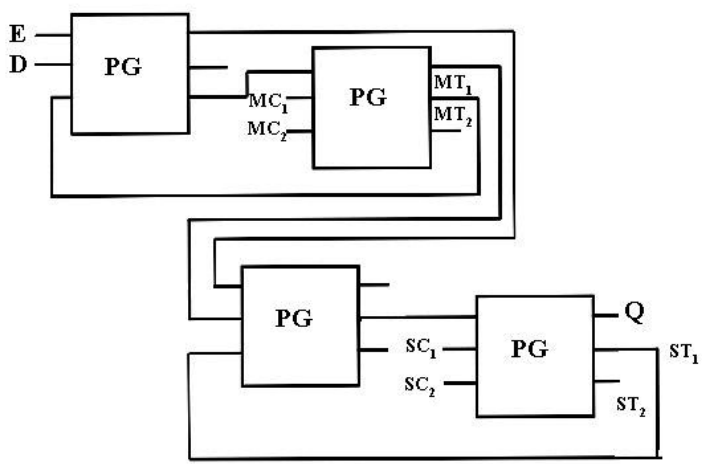

Figure 8: Design of Master slave D flipflops using Peres gate

Four Peres gate and Toffoli gate are used in master slave D Flip-flops. When enable is zero, the value of $d$ is passed to output $\mathrm{Q}$ otherwise it maintains the previous state. $\mathrm{mC} 1$, $\mathrm{mC} 2, \mathrm{SC} 1, \mathrm{SC} 2$ are the control modes for the master latch and slave latch. In normal mode, when enable and $\mathrm{d}$ value are one, output of $\mathrm{d}$ is passed to Q. In test mode, when all control signals are zero, when enable is one, the value of $d$ is reflected to the output q. Master slave flipflop is tested for two modes of operations.

\section{DESIGN Of DET FliPflops USING TOFFOLI AND Peres Gate}

Four Toffoli gates or Four Peres gates are used two Toffoli or Peres gate acts as master latch, while lower two Toffoli or Peres gate acts as a slave latch. $\mathrm{mC1}$ $\mathrm{mC} 2, \mathrm{sC} 1, \mathrm{sC} 2$ form the control signals for master latch and slave latch. Latch is tested under the fault free mode and test mode. Toffoli or Peres gate at the end acts as the 2:1 MUX. 


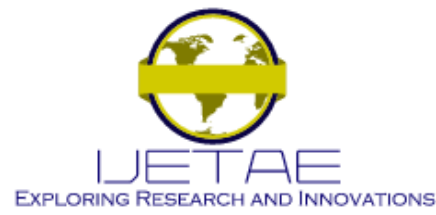

\section{International Journal of Emerging Technology and Advanced Engineering}

Website: www.ijetae.com (ISSN 2250-2459, ISO 9001:2008 Certified Journal, Volume 4, Issue 2, February 2014)

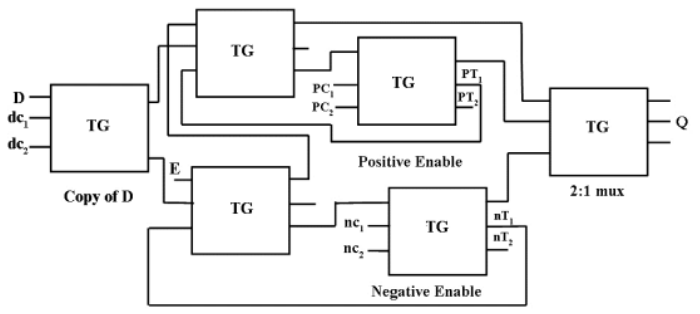

Figure 9: Design of DET flipflops using Toffoli gate

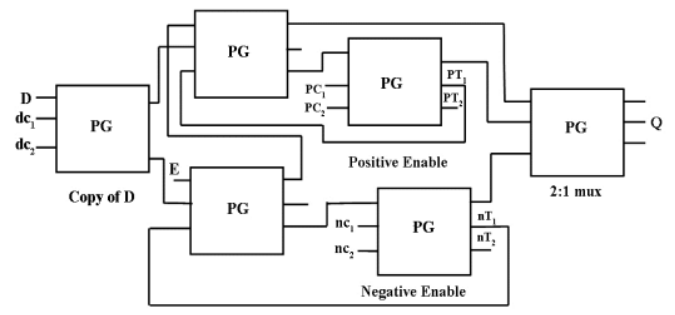

Figure 10: Design of DET flipflops using Peres gate

DET based Toffoli gate or Peres gate works in two modes operation. In normal mode, the value of nc1 and nc2 is 0 and 1 . In test mode, the value of pc1 and pc2 is either 0 or 1 . By setting all control signals as zero, when enable is one, the output $\mathrm{Q}$ is zero. When enable and $\mathrm{d}$ value are one, all control signals as zero, the output $\mathrm{Q}$ is one. Latch is tested for various combination of control signals and enable values.

\section{DESIGN OF TOFFOLI EQUIVALENT CIRCUIT}

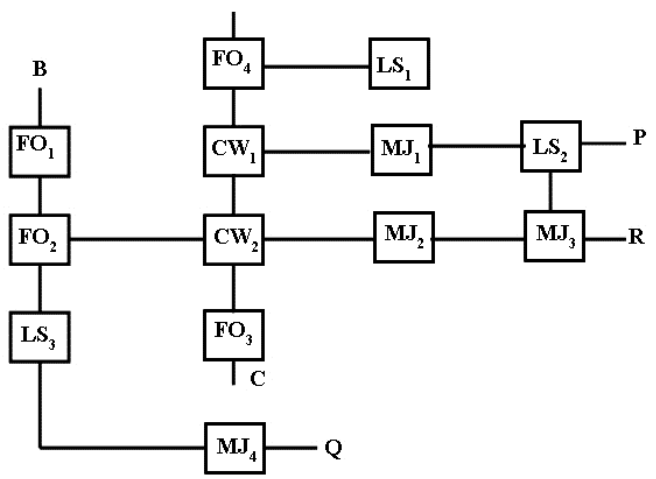

Figure 11: Toffoli equivalent circuit

In the existing work, equivalent circuit is drawn from QCA layout to obtain the maximum fault coverage. In this paper, the researchers propose the equivalent circuit obtained from Boolean expression of Toffoli gate.
Fanout (FO) are the components used in equivalent circuit. Cross wire (CW), L-shaped wire (LS), Majority voter $(\mathrm{MJ})$, are the components used in equivalent circuit. Cross wire $(\mathrm{CW})$ is to combine two variables by AND operation. Fanout (FO) is provided in order to drive the output. L-shaped wire (LS) perform the operation in which signal is passed from in and out. Majority voter (MJ) are provided to perform the AND operation. All components combined together in order to perform the maximum fault coverage.

\section{Design Of Peres Equivalent Circuit}

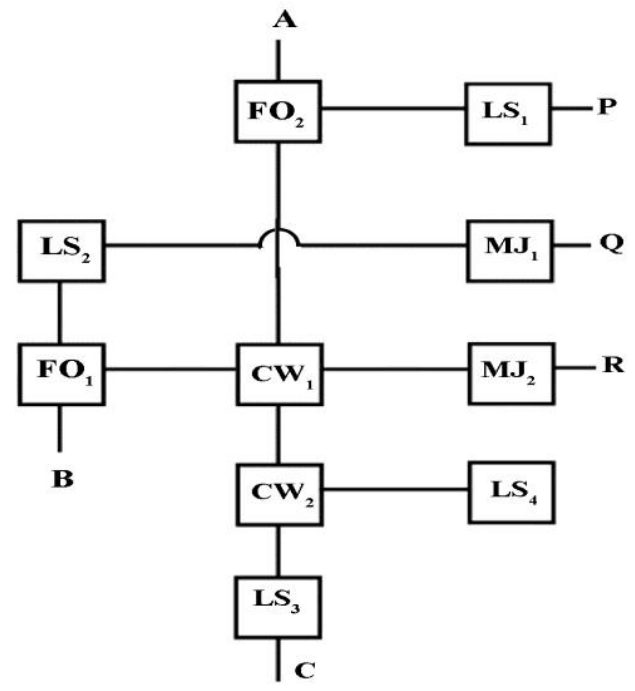

Figure 12: Peres equivalent circuit

In Existing work, MXCQCA and Fredkin gates are drawn in equivalent circuit, from QCA layout. In this paper, the researchers propose the equivalent circuit drawn from Boolean expression of Peres gate, Fan out (FO), Lshaped wire (LS), Majority voter (MJ), Cross wire (CW) are the components used. Crosswire $(\mathrm{CW})$ is to combine two variable by AND operation. Fanout $(\mathrm{FO})$ is provided to drive the output. Fanout is designed if the fault is 1, the output is inverted. L-shaped wire is to pass the signal from in and out, L-shaped wire is designed in such a way if fault is 1 , output is inverted. In Majority voter, the output is fault free if the fault is zero, otherwise stuck at fault exists. The output is tested for various combinations of faults in Toffoli gate and Peres gate. Faults are introduced to attain the maximum fault coverage. 


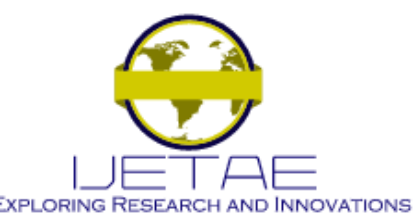

\section{International Journal of Emerging Technology and Advanced Engineering}

Website: www.ijetae.com (ISSN 2250-2459, ISO 9001:2008 Certified Journal, Volume 4, Issue 2, February 2014)

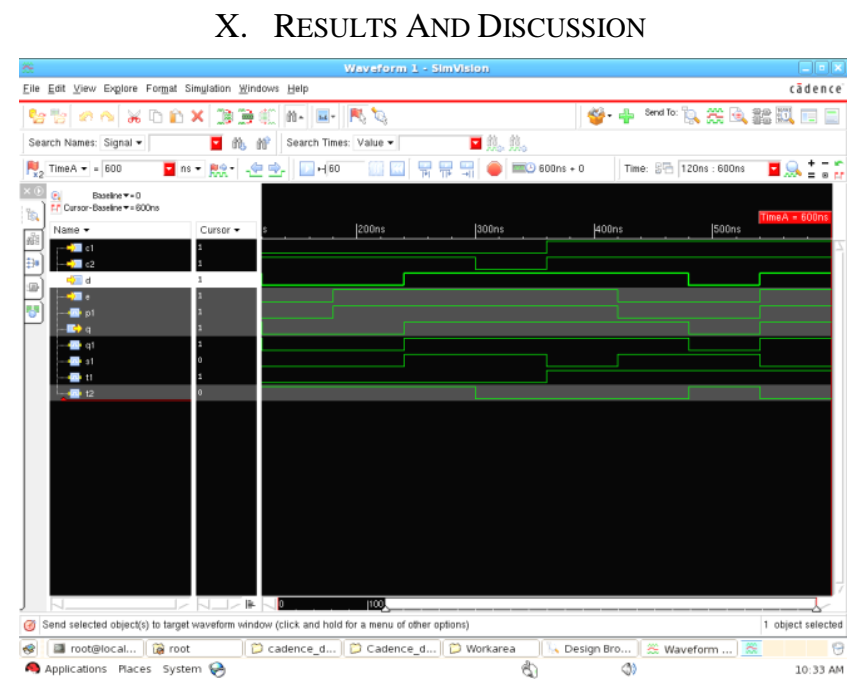

Figure 13: Output waveform of Reversible D latch with Control signals using Toffoli gate

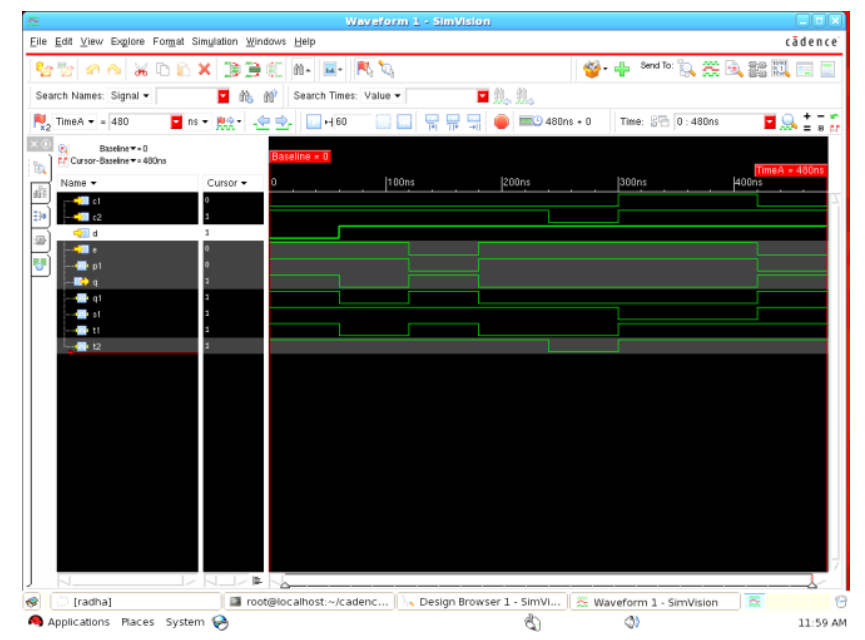

Figure 14: Output waveform of Reversible D latch with Control signals using Peres gate

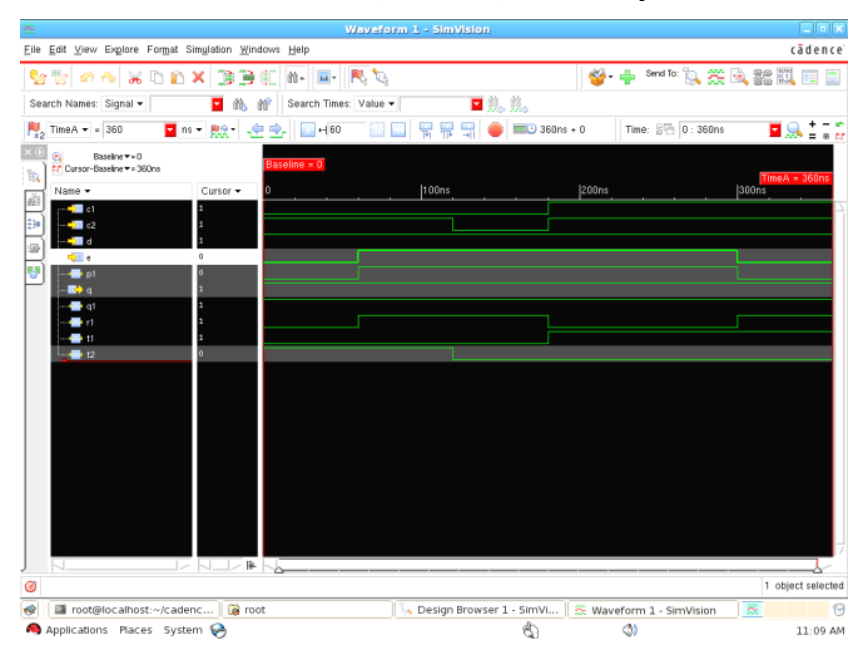

Figure 15: Output waveform of Negative enable Reversible D latch using Toffoli gate

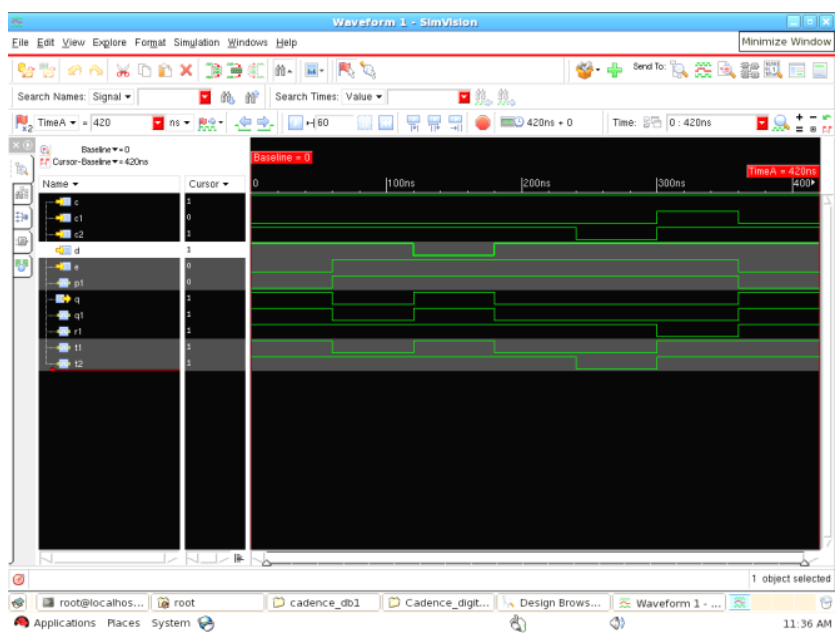

Figure 16: Output waveform of Negative enable Reversible D latch using Peres gate 


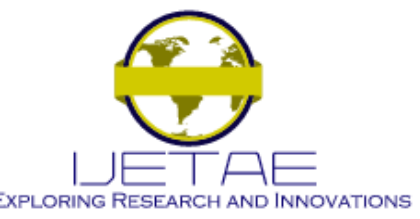

\section{International Journal of Emerging Technology and Advanced Engineering}

Website: www.ijetae.com (ISSN 2250-2459, ISO 9001:2008 Certified Journal, Volume 4, Issue 2, February 2014)

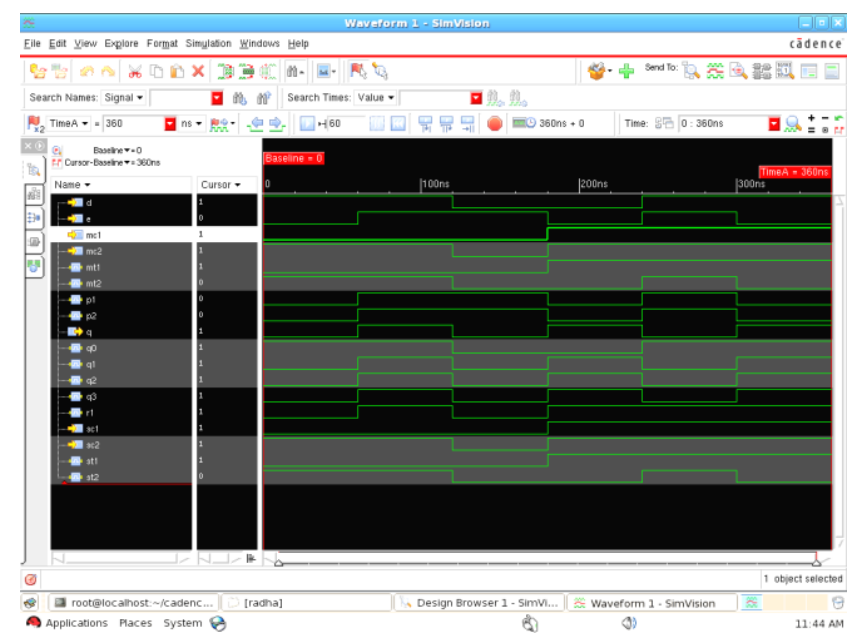

Figure 17: Output waveform of master slave D flipflop using Toffoli gate

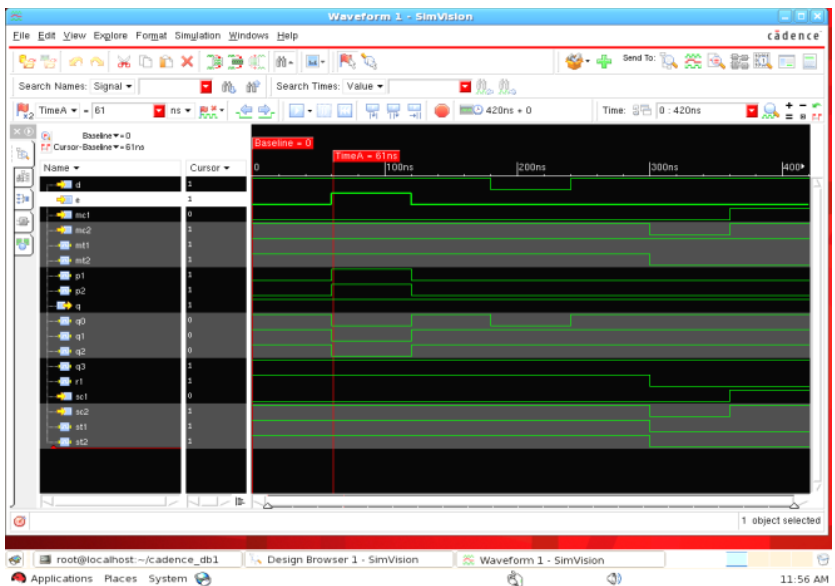

Figure 18: Output waveform of Master slave D flipflop using Peres gate

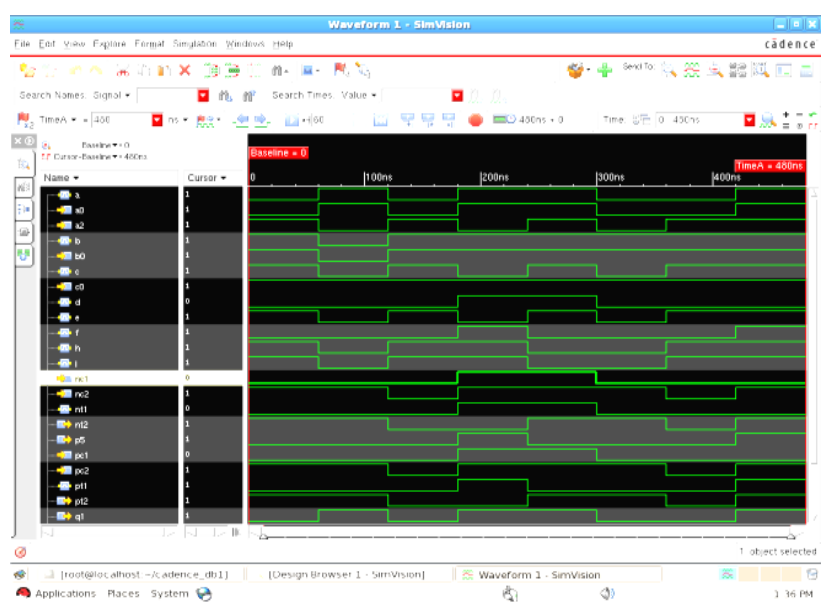

Figure 19: Output waveform of DET using Toffoli gate

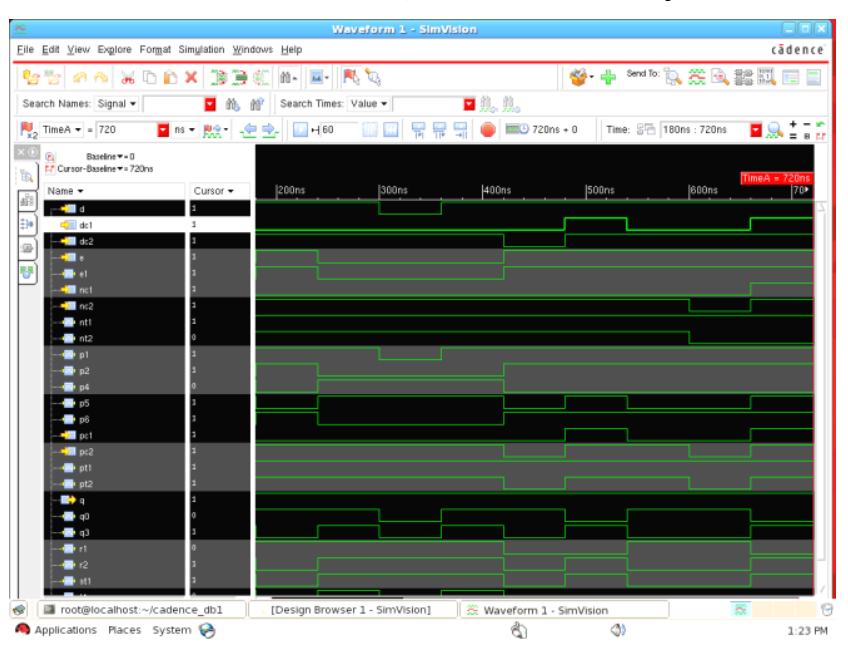

Figure 20: Output waveform of DET using Peres gate

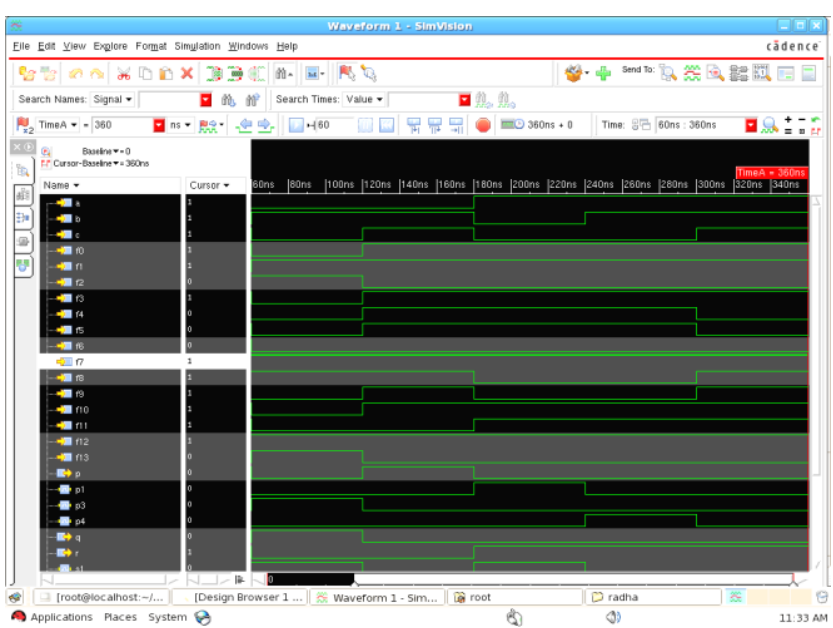

Figure 21: Output waveform of Toffoli equivalent circuit

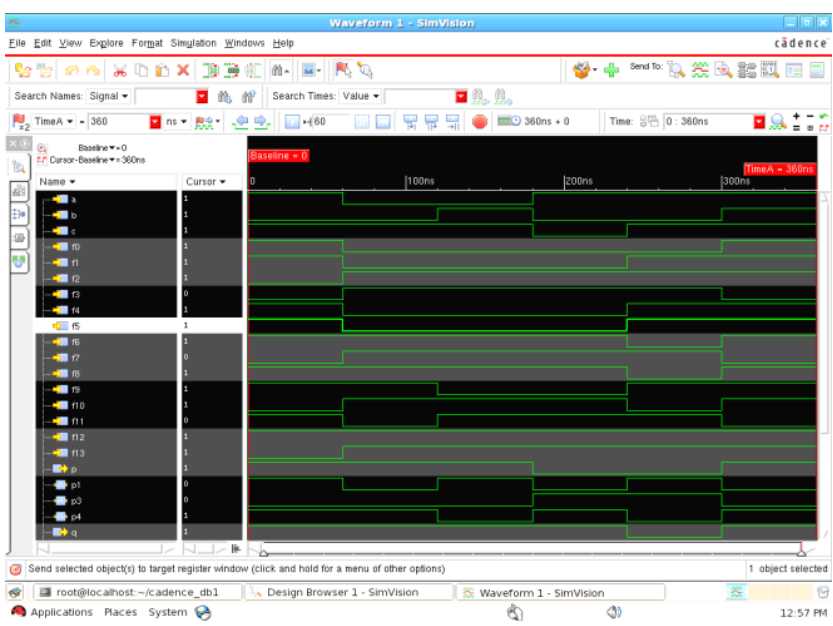

Figure 22: Output Waveform of Peres equivalent circuit 


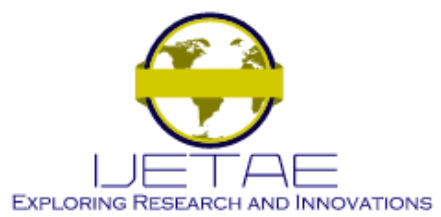

International Journal of Emerging Technology and Advanced Engineering

Website: www.ijetae.com (ISSN 2250-2459, ISO 9001:2008 Certified Journal, Volume 4, Issue 2, February 2014)

TABLE 3

EXISTING BLOCK AND PROPOSED BLOCK

\begin{tabular}{|l|l|l|l|}
\hline Parameters & $\begin{array}{c}\text { Existing } \\
\text { System } \\
\text { (Fredkin ) }\end{array}$ & $\begin{array}{c}\text { Proposed } \\
\text { System 1 } \\
\text { (Toffoli) }\end{array}$ & $\begin{array}{c}\text { Proposed } \\
\text { System 2 } \\
\text { (Peres) }\end{array}$ \\
\hline No Of Gates & 2 & 2 & 2 \\
\hline Area & 53 & 33 & 60 \\
\hline Timing & 88 & 118 & 98 \\
\hline
\end{tabular}

TABLE 4

EXISTING D LATCH CONTROL AND PROPOSED D LATCH CONTROL

\begin{tabular}{|l|l|l|l|}
\hline \multicolumn{1}{|c|}{ Parameters } & $\begin{array}{c}\text { Existing } \\
\text { System } \\
\text { (Fredkin ) }\end{array}$ & $\begin{array}{c}\text { Proposed } \\
\text { System 1 } \\
\text { (Toffoli) }\end{array}$ & $\begin{array}{c}\text { Proposed } \\
\text { System 2 } \\
\text { (Peres) }\end{array}$ \\
\hline No Of Gates & 4 & 2 & 1 \\
\hline Area & 93 & 33 & 27 \\
\hline Timing & 268 & 2 & 131 \\
\hline
\end{tabular}

TABLE 5

EXISTING NEGATIVE ENABLE D LATCH AND PROPOSED NEGATIVE ENABLE D LATCH

\begin{tabular}{|l|l|l|l|}
\hline Parameters & $\begin{array}{c}\text { Existing } \\
\text { System } \\
\text { (Fredkin ) }\end{array}$ & $\begin{array}{c}\text { Proposed } \\
\text { System 1 } \\
\text { (Toffoli) }\end{array}$ & $\begin{array}{c}\text { Proposed } \\
\text { System 2 } \\
\text { (Peres) }\end{array}$ \\
\hline No Of Gates & 4 & 3 & 1 \\
\hline Area & 93 & 67 & 27 \\
\hline Timing & 266 & 97 & 131 \\
\hline
\end{tabular}

TABLE 6

EXISTING MASTER SLAVE D FLIPFLOP AND PROPOSED MASTER SLAVE D FLIPFLOP

\begin{tabular}{|l|l|l|l|}
\hline Parameters & $\begin{array}{c}\text { Existing } \\
\text { System } \\
\text { (Fredkin ) }\end{array}$ & $\begin{array}{c}\text { Proposed } \\
\text { System 1 } \\
\text { (Toffoli) }\end{array}$ & $\begin{array}{c}\text { Proposed } \\
\text { System 2 } \\
\text { (Peres) }\end{array}$ \\
\hline No Of Gates & 7 & 2 & 5 \\
\hline Area & 160 & 33 & 100 \\
\hline Timing & 415 & 118 & 378 \\
\hline
\end{tabular}

TABLE 7

EXISTING DET FLIPFLOP AND PROPOSED DET FLIPFLOP

\begin{tabular}{|l|l|l|l|}
\hline Parameters & $\begin{array}{c}\text { Existing } \\
\text { System } \\
\text { (Fredkin ) }\end{array}$ & $\begin{array}{c}\text { Proposed } \\
\text { System 1 } \\
\text { (Toffoli) }\end{array}$ & $\begin{array}{c}\text { Proposed } \\
\text { System 2 } \\
\text { (Peres) }\end{array}$ \\
\hline No Of Gates & 9 & 11 & 5 \\
\hline Area & 213 & 193 & 100 \\
\hline Timing & 372 & 118 & 378 \\
\hline
\end{tabular}

TABLE 8

EXISTING EQUIVALENT CIRCUIT AND PROPOSED EQUIVALENT CIRCUIT

\begin{tabular}{|l|l|l|l|}
\hline Parameters & $\begin{array}{c}\text { Existing } \\
\text { System } \\
\text { (Fredkin ) }\end{array}$ & $\begin{array}{c}\text { Proposed } \\
\text { System 1 } \\
\text { (Toffoli) }\end{array}$ & $\begin{array}{c}\text { Proposed } \\
\text { System 2 } \\
\text { (Peres) }\end{array}$ \\
\hline No Of Gates & 50 & 16 & 18 \\
\hline Area & 888 & 316 & 336 \\
\hline Timing & 1220 & 586 & 586 \\
\hline
\end{tabular}




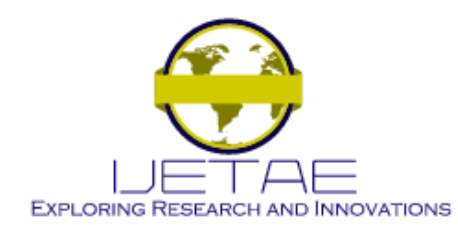

\section{International Journal of Emerging Technology and Advanced Engineering \\ Website: www.ijetae.com (ISSN 2250-2459, ISO 9001:2008 Certified Journal, Volume 4, Issue 2, February 2014)}

In Figure 13 and Figure 14, output is tested for D latch by giving enable signal and analyzed the operation of

Two modes

In Figure 15 and Figure 16, output is tested for negative enable $\mathrm{D}$ latch by giving enable and $\mathrm{d}$ values.

In Figure 17 and Figure 18, output describes how Master and slave circuits works by giving enable values and tested for two modes of operation.

In Figure 19 and Figure 20, output is tested for fault free mode and test mode by giving control signal values. In Figure 21 and Figure 22, output is tested for 13 fault values, and analyzed fault in each component.

Table 3 summarizes the Area, No of gates and Timing in Existing system is better than proposed system.

Table 4 describes the Area, No of gates and Timing in Existing system is better than proposed system

Table 5 demonstrates the Area, No of gates and Timing in Existing system is better than proposed system

Table 6 analyzes the Area, No of gates and Timing in Existing system is better than proposed system

Table 7 summarizes the Area, No of gates and Timing in Existing system is better than proposed system

Table 8 describes the Area, No of gates and Timing in Existing system is better than proposed system

\section{CONCLUSION}

This paper describes the design of reversible sequential circuits based on two gates, namely Toffoli gate and Peres gate. We have proposed the equivalent Circuit for Toffoli gate and Peres gate in order to less area, computation time and maximum fault coverage. Area, number of gates and timing are analyzed in these gates. Toffoli gate is better in terms of power consumption compared to fredkin gate. In proposed work, Area, number of gates and timing are reduced compared to existing work. In future work, Ultra low power memory circuits and Digital circuits can be designed.

\section{REFERENCES}

[1] Dmitri Maslov, Gerhard W.Dueck, and D.Micheal miller,'Toffoli network synthesis with Templates", 2004

[2] Vivek V.shende,Aditya K.Prasad,Igor L.Markov and John P.Hayes,"Synthesis of reversible logic circuits",IEEE trans,Vol.22.,No.6.,Jun 2003

[3] Himanshu Thapiyal and M.B.Srinivas,"A Beginning in the Reversible logic synthesis of sequential circuits", IEEE trans, 2005

[4] J.E.Rice,"The State of reversible logic synthesis", sep 2005.

[5] Sivakumar sastry hari,shyam shroff,sk.Noor Mahammad and v.Kamakoti","Efficient building blocks for sequential circuit design",IEEE trans,2006.

[6] Pallav gupta,Abhinav Agrawal and Niraj k.Jha,"An Algorithm for synthesis of reversible logic circuits",IEEE trans ,Vol.25.,No.11.,Nov 2006.

[7] Dilip P.Vasudevan,Parag k.lala,Jia Dia,J.Patrick Parkerson,"Reversible logic design with online testability",IEEE trans ,Vol.55.,No.2,Apr 2006.

[8] Min-Lun Chuang, Chun-Yao Wang, "Synthesis of reversible sequential elements", IEEE trans, 2007

[9] Himanshu Thapiyal, Nagarajan Ranganathan,"Reversible logic based concurrently testable latches for molecular QCA", IEEE trans., vol.59, No.1,Jan 2010.

[10] Sk.Noor Mahammed and Kamakoti veezhinathan,"Constructing online testable circuits using reversible logic", IEEE trans., vol.59, No.4. Dec 2010

[11] Himanshu Thapiyal and Nagarajan Ranganathan,"Design of reversible sequential circuits optimizing quantum cost, delay and garbage outputs", ACM Journal on emerging tech in computer Systems, vol.6, No.4,Dec 2010.

[12] Lafifa Jamal,Md.shamsujjoha, and Hafiz Md.Hasan babu,” Design of optimal reversible carry look ahead adder with optimal garbage and quantum cost "International Journal of Engineering and technology, vol.2.,No.1.,Jan 2012

[13] Bhagya lakshmi HR, Venkatesha MK,"Design of sequential circuit elements using reversible logic gates", International E-conference on Information Technology and Applications, vol.2, May 2012.

[14] Sheenu Thapar, Parminder kaur, Nidhi syal,'Multiplier circuit based on reversibility principle", An International Journal (ESTIJ), vol.2, No.4, Aug 2012.

[15] Himanshu Thapliyal,Nagarajan Ranganathan,'Design of Testable Reversible Sequential Circuits"IEEE trans,Vol.21.,No.7,July 2013

[16] Raghave garipelly, p.Madhu kiran, A.Santhosh kumar,"A Review on reversible logic gates and their implementation", International Journal of emerging Technology \& Advanced Engineering, vol.3.,Mar 2013. 